Mots. Les langages du politique

\title{
Le discours politique n'est pas transparent. Permanence et transformations d'un objet de recherche
}

Paul Bacot, Marlène Coulomb-Gully, Jean-Paul Honoré, Christian Le Bart, Claire Oger et Christian Plantin

\section{OpenEdition}

\section{Journals}

Édition électronique

URL : https://journals.openedition.org/mots/19841

DOI : $10.4000 /$ mots. 19841

ISSN : 1960-6001

Éditeur

ENS Éditions

Édition imprimée

Date de publication : 30 novembre 2010

Pagination : 5-9

ISBN : 978-2-84788-235-3

ISSN : 0243-6450

Référence électronique

Paul Bacot, Marlène Coulomb-Gully, Jean-Paul Honoré, Christian Le Bart, Claire Oger et Christian Plantin, «Le discours politique n'est pas transparent. Permanence et transformations d'un objet de recherche », Mots. Les langages du politique [En ligne], 94 | 2010, mis en ligne le 17 décembre 2010, consulté le 28 avril 2022. URL : http://journals.openedition.org/mots/19841 ; DOI : https://doi.org/ $10.4000 /$ mots. 19841 


\section{Mots \\ Les langages du politique}

№ 94 novembre 2010

\section{Trente ans d'étude des langages du politique (1980-2010)}

ouvrage coordonné par Paul BACOT, Marlène Coulomb-GulLY, Jean-Paul Honoré, Christian Le BART, Claire Oger, Christian Plantin

\section{SOMMAIRE}

Paul Bacot, Marlène Coulomb-Gully, Jean-Paul Honoré, Christian Le Bart, Claire Oger, Christian Plantin

Le discours politique n'est pas transparent. Permanence

et transformations d'un objet de recherche

OUTILS ET ENJEUX DU DISCOURS POLITIQUE

Ruth Amossy, Roselyne Koren

Argumentation et discours politique

Christian Plantin

Argumentation-rhétorique. Les eaux mêlées

Caroline Ollivier-Yaniv

Discours politiques, propagande, communication, manipulation 
Marc Bonhomme

La caricature politique

Paul Bacot

Développement et diversification d'une onomastique politique

Ruth Wodak

The Discursive Construction of History. Brief Considerations

Henri Boyer

Les politiques linguistiques

\section{LIEUX DE LA PRODUCTION DU DISCOURS POLITIQUE}

Christian Le Bart

Parler en politique

Dominique Maingueneau

Le discours politique et son «environnement»

Alice Krieg-Planque, Claire Oger

Discours institutionnels. Perspectives pour les sciences

de la communication

Sophie Béroud, Josette Lefèvre

Le corpus syndical. Une expérience au long cours

Corinne Gobin, Jean-Claude Deroubaix

L'analyse du discours des organisations internationales.

Un vaste champ encore peu exploré

François de la Bretèque

Le retour de la parole politique dans le cinéma français

Jean-Claude Soulages

Vie et mort du citoyen cathodique

Jacques Guilhaumou

Les discours de la Révolution française.

Aperçu d'ensemble d'un trajet de recherche (1980-2009) 


\section{DISCIPLINES ET CHAMPS DE RECHERCHE POUR L'ÉTUDE DES LANGAGES DU POLITIQUE}

Philippe Braud

L'apport de la science politique à l'étude des langages du politique

Claire Blandin

L'apport de l'histoire des médias à l'étude des langages du politique

Jean-François Tétu, Bernard Lamizet

Les SIC et les langages du politique

Sylvianne Rémi-Giraud

Sémantique lexicale et langages du politique.

Le paradoxe d'un mariage difficile?

Marlène Coulomb-Gully, Juliette Rennes

Genre, politique et analyse du discours.

Une tradition épistémologique française gender blind

Johannes Angermüller

Analyser le discours politique en Allemagne (1980-2010)

Érik Neveu

L'apport de Pierre Bourdieu à l'analyse du discours.

D'un cadre théorique à des recherches empiriques

Roselyne Ringoot

Questionner le discours avec Michel Foucault.

Actualisations théoriques et actualité éditoriale

\section{ENTRETIEN}

Maurice Tournier

Mots et politique, avant et autour de 1980

La revue Mots. Les langages du politique encourage l'usage des rectifications de l'orthographe proposées par le Conseil supérieur de la langue française et approuvées par l’Académie (Journal officiel, $n^{\circ}$ 100, 6 décembre 1990). 


\section{Paul Bacot, Marlène Coulomb-Gully, Jean-Paul Honoré, Christian Le Bart, Claire Oger, Christian Plantin}

\section{Le discours politique n'est pas transparent. Permanence et transformations d'un objet de recherche}

Peut-on dire que Mots. Les langages du politique a trente ans? Rien n'est moins sûr. À l'automne 1980, paraît en effet la première livraison d'une publication qui porte pour titre l'acronyme Mots, dont le développement est « Mots, Ordinateurs, Textes, Sociétés». Ce n'est que neuf ans plus tard que notre revue prendra son nom actuel. Mais seuls des passionnés du discours feront preuve de tels scrupules onomastiques : notre revue a bien trente ans d'âge! L'équipe rédactionnelle restait la même et l'éditeur était encore les Presses de la Fondation nationale des sciences politiques ${ }^{1}$. L'objet s'était seulement élargi - on était passé de l'analyse du seul lexique à celle de toutes les formes de langage et précisé - la dimension proprement politique des productions étudiées était clairement affichée. Créée à Saint-Cloud et dirigée par Maurice Tournier seul jusqu'en 2000, puis avec Pierre Fiala jusqu'en 2002 et par celui-ci jusqu'en 2005, elle est la seule revue francophone spécifiquement dédiée à l'étude des langages du politique - à tous les modes de construction symbolique (y compris la chanson, le cinéma, la caricature, la théâtralisation, le protocole, les rites...) d'un politique entendu largement.

Pour célébrer cet anniversaire, il fallait assurément un numéro spécial, mais nous avons très vite écarté l'idée que celui-ci pût ne porter que sur l'histoire de

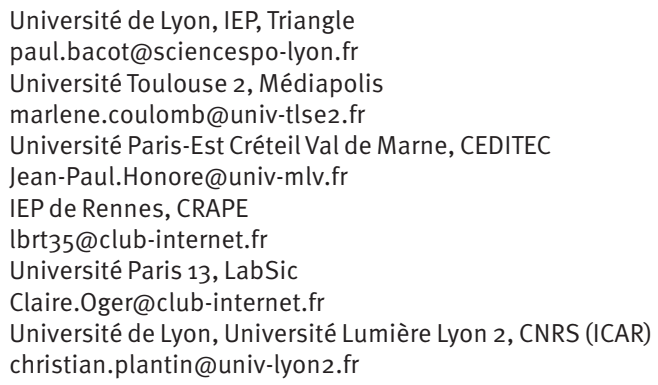

1. En l'an 2000, c'est ENS Éditions qui a repris le titre. 
cette revue aujourd'hui trentenaire, qui l'aurait certes bien mérité mais que nous avons préféré honorer autrement. Nous avons voulu saisir l'occasion qui nous était donnée pour tenter un bilan de trente années d'études des langages du politique - dans les pages de la revue, nécessairement, mais aussi bien au-delà. Pour ce faire, nous avons demandé à trente chercheurs, français et étrangers, tous reconnus dans leurs spécialités, d'apporter leur contribution à ce travail. Quel était l'état de ce champ scientifique en 1980 ? Comment a-t-il évolué depuis lors? Quelles institutions, quelles disciplines, quelles écoles, quels auteurs ontils marqué ce cheminement? Que savons-nous aujourd'hui en la matière que nous ignorions il y a trente ans? Quels sont les débats en cours et les questionnements actuellement privilégiés ? Quels sont les chantiers ouverts ou à ouvrir? Quelles sont les limites et les obstacles épistémologiques ou méthodologiques rencontrés dans cette entreprise? L'ensemble des textes ainsi recueillis ne peut assurément prétendre à une quelconque exhaustivité. Notre espoir est seulement qu'il pourra fournir à tous ceux qui s'intéressent au langage ou au politique, et a fortiori aux usages du langage en politique, des repères factuels et des points d'appui pour leurs propres réflexions.

Mais comme nous avons la faiblesse de penser que l'apport de Mots. Les langages du politique a été central dans cette histoire intellectuelle, nous avons demandé à son fondateur de revenir sur les conditions de sa création : l'entretien avec Maurice Tournier qui clôt ce volume est un document d'une grande richesse et d'une rare érudition. Il permet de mieux comprendre par quels croisements disciplinaires, épistémologiques et méthodologiques s'est construit un champ scientifique aujourd'hui reconnu comme tel, mais aussi dans quelle conjoncture spécifique, tant politique qu'académique, et avec quels outils innovants de telles avancées ont pu voir le jour.

De la lecture des pages qui suivent se dégagent quelques grandes lignes de force, au-delà de la diversité des approches et des positionnements de leurs auteurs. Elles concernent notamment la relation de l'analyse des langages du politique avec ses environnements social et scientifique, mais aussi les motivations des chercheurs concernés et, partant, les objets qu'ils ont privilégiés. Ces quelques considérations souffriront nécessairement du caractère partiel du tableau constitué par les contributions ici rassemblées (elles privilégient les productions francophones, sans ignorer évidemment le reste du monde et la circulation internationale des problématiques et des connaissances acquises). Mais elles peuvent s'apparenter à quelque chose comme un rapport d'étape. Tentons donc de l'esquisser.

D'abord, l'environnement social, et notamment politique. Il est impossible, dans les années quatre-vingt, de faire abstraction de la proximité encore perceptible de la seconde guerre mondiale et des guerres coloniales, et de la réalité très sensible de la Guerre froide et des bouleversements institutionnels et sociétaux des Trente glorieuses : la télévision, l’immigration postcoloniale et 
l'avènement de la Cinquième République pour ce qui est de la France (les élections présidentielles deviennent vite un objet de forte investigation). De même, bien sûr, Mai 1968 et les grands mouvements idéologiques qui précèdent et suivent ces événements (le féminisme, le tiers-mondisme et l'écologie, notamment) fournissent une véritable toile de fond au développement des interrogations sur la dimension langagière du politique, en même temps que d'une certaine façon ils contribuent à le rendre possible.

Ensuite, l'environnement scientifique, qu'il s'agisse des théories et des concepts, ou des méthodes et des techniques. L'analyse du discours politique est bien sûr d'abord redevable aux sciences du langage, mais aussi à l'histoire sociale, à la philosophie et à la statistique, et dans une mesure moindre et plus récemment, à la sociologie, à l'anthropologie et à la science politique. Les naissantes sciences de l'information et de la communication apporteront un peu plus tard leur contribution. L'interdisciplinarité, au cœur de l'identité de la revue, est souvent interrogée, qui se heurte à trois obstacles principaux: le coût que représente pour les spécialistes d'une science l'appropriation des outils d'une autre science; l'incertaine adéquation des instruments de l'une aux attentes de l'autre; l'effet des découpages académiques.

On peut repérer quelques noms de grands auteurs, dont l'impact sur les études ici évoquées apparaît de manière récurrente. Citons notamment, audelà d'Aristote et en dehors des linguistes (Benveniste ou Bakhtine plus que Saussure), Louis Althusser, Pierre Bourdieu, Michel Foucault, Jürgen Habermas, Michel Pêcheux, mais aussi Erving Goffman ou Roland Barthes. Le marxisme, le structuralisme, la phénoménologie, l'interactionnisme, notamment, l'ethnométhodologie plus marginalement, ont fourni outils et concepts. Et l'on ne sera pas surpris de voir l'analyse se complexifier au fil des ans, faisant une plus large part à l'acteur individualisé, mais aussi au social incorporé et objectivé. Mais le cœur du programme reste le même : remettre en cause la transparence du discours, par la prise en compte «non seulement de ce qui est dit, mais de la manière, plus signifiante souvent, dont cela est dit »². Et pour ce faire, la quantification et le traitement automatique de corpus seront fondateurs - nous avons commencé cette présentation en rappelant le premier nom de la revue et Maurice Tournier revient longuement, dans son entretien, sur l'aventure de la lexicométrie, dont l'histoire quarantenaire du corpus syndical reste emblématique.

Les motivations des chercheurs se lisent d'une certaine façon dans le choix des objets étudiés. Il s'agit souvent de dévoiler, et partant de dénoncer - le totalitarisme, le colonialisme, le racisme, l'antisémitisme, le sexisme, les extrémismes, la xénophobie, ou plus simplement l'erreur, la fallace, la mauvaise foi, la langue de bois ou le discours présenté comme «expert»: le propos

2. Maurice Tournier, Mots. Les langages du politique, nº18, mars 1989, p. 3 . 
est souvent critique, sans cesser d'être scientifique. Du coup, le politique semble ramené à un affrontement entre un pouvoir a priori manipulateur et des dominés potentiellement révoltés - un peuple authentique que font parler des leaders d'organisations et des représentants élus : on comprend alors mieux la place du discours révolutionnaire dans les travaux à l'origine de la revue, ou plus tard celle des études interrogeant l'efficacité des discours militant, médiatique ou gouvernemental, et la performativité des politiques publiques - politiques linguistiques ou mémorielles, par exemple.

D’autres travaux, pourtant, vont au-delà du discours politique défini par le statut du locuteur, au-delà de la politique incarnée par des professionnels cherchant à dominer les citoyens - par l'imposition, la séduction ou la conviction - ou à parler en leur nom. Le politique est alors compris comme une lutte entre représentations concurrentes de la conflictualité sociale, entre mises en scène du consensus et du dissensus. D'autres locuteurs peuvent alors être pris en considération, mais aussi d'autres genres de discours, d'autres prises de parole, d'autres supports ou dispositifs : si, il y a trente ans, l'imprimé était l'objet de la plupart des travaux, l'audiovisuel retient progressivement de plus en plus l'attention, et aujourd'hui les sites et les blogs sont traités comme des vecteurs majeurs du propos politique. Mais finalement, tous les analystes du discours politique ont toujours travaillé sur l'argumentation entendue au sens large et, au-delà, sur la bataille pour la légitimation des personnes et des institutions, des idées et des actes - bataille porteuse d'un système de contraintes s'exerçant sur l'énonciation. En la matière, si le pouvoir des mots ne fait aucun doute, la question de son fondement reste objet de débats.

Un autre fil conducteur peut être suivi dans la lecture de ce volume : celui du choix de l'objet politique pour traiter du discours - et réciproquement. On se demandera si le discours politique se prête particulièrement bien aux analyses rhétoriques, étant peuplé de métaphores, de métonymies, d'emprunts, de formules, de slogans, mais aussi de noms propres. On ne peut l'entendre qu'en prenant en compte le contexte, l'interdiscours, les références, les connotations, la polysémie, voire la gestuelle coverbale. Ce ne serait donc pas par hasard si l'analyse du discours s'est développée principalement autour de textes politiques, tout comme bien avant elle les études de rhétorique. Mais si l'inverse peut sembler logiquement évident - l'importance du discours dans l'analyse politologique -, la vérité oblige à dire que la science politique ne porte aux langages qu'un intérêt apparemment limité et relativement récent, malgré les écrits précurseurs de Frédéric Bon3. D’une manière générale, les principales disciplines qui s’intéressent au politique n'accordent aux approches langagières qu'une place réduite, mais en développement certain.

3. Frédéric Bon, «Langage et politique », Traité de science politique, M. Grawitz, J. Leca éd., Paris, PUF, 1985, t. 3, p. 537-573. 
Tout discours serait-il politique, et le politique se résoudrait-il en discours? Le lecteur des pages qui suivent ne sera parfois pas loin de le penser. Nous préférerons dire ici que l'objectif aujourd'hui commun aux chercheurs évoqués ci-après est de débusquer le politique dans le langage, et le langage dans le politique. 